1998-03-01

\title{
Breakpoint Skeletal Representation and Compression of Document Images
}

\author{
William A. Barrett \\ william_barrett@byu.edu \\ Bryan S. Morse \\ morse@byu.edu
}

Eric N. Mortensen

Follow this and additional works at: https://scholarsarchive.byu.edu/facpub

Part of the Computer Sciences Commons

\section{Original Publication Citation}

D. Tam, W. Barrett, B. Morse, and E. Mortensen, "Breakpoint skeletal representation and compression of document images," in Proceedings Data Compression Conference, March 1998.

\section{BYU ScholarsArchive Citation}

Barrett, William A.; Morse, Bryan S.; and Mortensen, Eric N., "Breakpoint Skeletal Representation and Compression of Document Images" (1998). Faculty Publications. 648.

https://scholarsarchive.byu.edu/facpub/648 


\title{
Breakpoint Skeletal Representation and Compression of Document Images
}

\author{
David Tam*, William Barrett ${ }^{\dagger}$, Bryan Morse, and Eric Mortensen \\ Department of Computer Science \\ Brigham Young University, Provo, Utah 84604 \\ *kwt@python.cs.byu.edu, † barrett@cs.byu.edu
}

We present a new method for representation and (lossy) compression of bitonal document images. The technique extracts a skeletal medial axis from each object using a true Euclidean distance map of the image and then finds piecewise linear breakpoints in the skeleton to create a breakpoint skeletal representation, bps, (Fig. 1). The bps is encoded for each object as a set of triples $\left\{\left\langle x_{1}, y_{1}, r_{1}\right\rangle,\left\langle\Delta x_{2}, \Delta y_{2}, \Delta r_{2}\right\rangle, \ldots\left\langle\Delta x_{n}, \Delta y_{n}, \Delta r_{n}\right\rangle\right\}$ where $\left\langle x_{1}, y_{1}, r_{l}\right\rangle$ contains the coordinate and distance (radius, $r_{l}$ ) of the initial breakpoint from the closest point on the perimeter of the object and $\left\langle\Delta x_{i}, \Delta y_{i}, \Delta r_{i}\right\rangle$ represents the difference in location and radius between the current and the previous breakpoint for the remaining $n-1$ breakpoints.

The original binary object is reconstructed by first reconstructing the skeleton using linear interpolation between breakpoints and then fractionally dilating each point on the skeleton with the (linearly interpolated) radius, $r_{i}$. For noninteger $r_{i}$ fractional dilation provides a natural antialiasing in the reconstructed image.

Breakpoints can be extracted to preserve fine detail or a more coarse representation by tightening or relaxing the pruning radius respectively. If, in extracting breakpoints, the pruning radius is set to zero, the reconstruction is almost lossless, but the compression is worse. Increasing the pruning radius eliminates noise due to sampling and pixelization of the original document, and coupled with fractional dilation, reconstructs the original pattern more cleanly than when it was scanned (Fig. 2). Increasing the radius too much, however, may result in the loss of important object detail, particularly for small objects.

bps compression compares favorably (by a factor of up to 6) with CCITT Group 3 and JBIG for certain classes of images (fewer and larger objects, Fig. 3). However, for images containing many small objects, bps performs worse because of a relatively larger number of breakpoints per object with shorter segments between breakpoints. With economical support for nonlinear segments and consequently fewer breakpoints, or incorporation of skeletal shape primitives, bps compression could be improved significantly.

Advantages of the bps representation are (1) It preserves object structure for subsequent analysis and recognition. (2) It preserves locality (each object can be reconstructed separately). (3) It is scalable - objects can be reconstructed to any size for multi-resolution display and browsing. (4) Skeletal segments can be hierarchically ordered both within and between objects to provide variable level of detail in browsing and speedup in transmission and download. (5) Support of object structure provides a higher level representation with the potential for higher level compression through the use of pattern matching and substitution techniques.

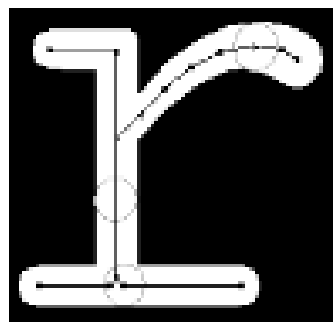

Fig. 1 Breakpoint Skeletal Representation

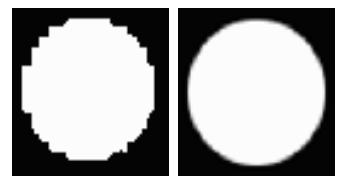

Fig. 2 Fractional Dilation provides natural antialiasing in reconstruction.
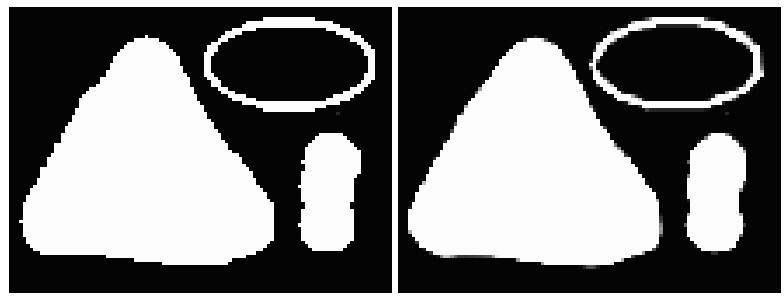

Fig. 3 Original image (left), bps (right) 